Evaluation of a freeze-clamping technique designed for two- and three-dimensional metabolic studies of rat liver in vivo. Quenching efficiency and effect of clamping on tissue morphology.

Quistorff, Bjørn; Poulsen, Hemming

Published in:

Analytical Biochemistry

Publication date:

1980

Document version

Publisher's PDF, also known as Version of record

Citation for published version (APA):

Quistorff, B., \& Poulsen, H. (1980). Evaluation of a freeze-clamping technique designed for two- and threedimensional metabolic studies of rat liver in vivo. Quenching efficiency and effect of clamping on tissue morphology. Analytical Biochemistry, 108(2), 249-256. 


\title{
Evaluation of a Freeze-Clamping Technique Designed for Two- and Three-Dimensional Metabolic Studies of Rat Liver in Vivo. Quenching Efficiency and Effect of Clamping on Tissue Morphology
}

\author{
BJørn Quistorff† ANd Hemming Poulsen
}

\begin{abstract}
Department of Biochemistry A, University of Copenhagen, and Department of Pathology, Hvidovre Hospital, University of Copenhagen, Copenhagen, Denmark
\end{abstract}

Received April 10, 1980

\begin{abstract}
In order to apply a previously described freeze-clamping technique (B. Quistorff and B. Chance, 1980, Anal. Biochem. 108, 237-248) in three-dimensional metabolic studies, it is necessary to clarify to which extent tissue morphology as well as metabolic state is preserved in the part of the freeze-clamped sample used for such studies. The present paper reports a comparative histological examination of rat liver, either freeze-clamped, applying the technique mentioned, or frozen without compression. It is demonstrated that neither the intra- nor the interlobular morphology of the central part of the sample is disturbed by the freeze-clamping process. In clamped as well as unclamped liver samples portal tracts could be identified, i.e., distinguished from efferent veins, in a depth below the capsule of $100-200 \mu \mathrm{m}$. Measurements of ATP, ADP, AMP, and $P_{i}$ in the freeze-clamped sample at increasing distance from the surface indicate that for in situ freezing, significant metabolic changes did not occur until a depth of about $1 \mathrm{~mm}$, while a delay of freezing of about 6 s seems to cause metabolic changes in the entire sample.
\end{abstract}

In a preceding paper (1) a technique was described for freeze-clamping and lowtemperature milling of the frozen sample for the purpose of three-dimensional metabolic studies in vivo. It was realized that the freeze-clamping step in two respects was crucial for the reliability of the threedimensional studies later to be performed on these samples: first, the possibility of distortion of the tissue architecture in the frozen sample, and second, the efficiency of the freeze-stop of metabolic processes in different parts of the sample.

This communication gives a histological evaluation of tissue architecture of freezeclamped rat liver, judging the integrity of the inter- and intralobular morphology. In addition, consecutive sections of the frozen liver sample, obtained by low-

† Correspondence to: Bjørn Quistorff, Department of Biochemistry A, Blegdamsvej 3, 2200 Copenhagen, Denmark. temperature milling (1) are analyzed for the content of a number of labile metabolites in order to establish the degree to which the in vivo metabolic state is preserved in different parts of the sample.

\section{MATERIALS AND METHODS}

\section{Animals}

Fed, male Wistar rats, weighing 250$300 \mathrm{~g}$ were used. Experiments were performed 20-30 min following sodium pentobarbital anaesthesia, $75 \mathrm{mg} / \mathrm{kg}$ ip.

\section{Histological Evaluation of the Effect of \\ Freeze-Clamping on Tissue Morphology}

A liver lobe was freeze clamped in situ as previously described (1) (Sample I). Another lobe from the same liver was excised and frozen without compression being placed on an aluminum disk precooled in liquid nitrogen (Sample II). From the 
central part of both samples, a block of about $6 \times 6 \times 2 \mathrm{~mm}$ was cut out in the frozen state applying a low-temperature saw (instrument II (1)). Each block was divided in two parts. One part was subjected to formaldehyde fixation (phosphatebuffered formaldehyde, $\mathrm{pH} 7.0$ (2)) and paraffin mounting for serial sectioning. The other part was freeze sectioned (2). Series of 50 to 300 consecutive sections of a thickness of $4 \mu \mathrm{m}$ were cut from each specimen. The procedure described above was carried out on livers from six different animals, three on which histological sections were cut parallel to the surface of the liver and three cut perpendicular. All sections were stained with chromotrope aniline blue (CAB) ${ }^{1}$ (3).

\section{Metabolic Evaluation of the \\ Freeze-Clamping and the Milling \\ Process}

Tissue sampling. There were two groups of animals, A and B, with five animals in each group. Applying the technique described in (1), a liver lobe was freeze clamped in situ, group A, while in group B the lobe was excised and freeze clamped 5-7 s later. The samples were transferred to a glove box at $-25^{\circ} \mathrm{C}$ in which a low-temperature milling instrument was operated (instrument II in (1)). The first $50-100 \mu \mathrm{m}$ of all samples was milled off and the powder discarded. On the samples from both groups the following experiment was performed.

The freeze-clamped sample was milled a number of times with cuts parallel to the surface of the sample, each cut of a thickness of 100-200 $\mu \mathrm{m}$. The milling powder of each cat was collected separately, weighed, and extracted as described below. The thickness of the total tissue layer cut off was $2-3 \mathrm{~mm}$.

Analytical technique. All samples were weighed and extracted in the glove box at $-20^{\circ} \mathrm{C}$ as described previously (1). Standard

\footnotetext{
blue.
}

enzymatic metabolite assays for adenine nucleotides and inorganic phosphate were performed on the neutralized tissue extracts. ATP was measured spectrophotometrically, ADP and AMP fluorometrically according to (4), and inorganic phosphate $\left(P_{\mathrm{i}}\right)$ according to $(5)$. Internal standards, added to the methanol $/ \mathrm{HCl}$ extracts were recovered by $96-107 \%$.

Statistical evaluation of the data of Tables 1 and 2 were based on paired data $t$ tests, performed on the raw data.

\section{RESULTS}

\section{Histological Evaluation of the Effect of Freeze-Clamping on Liver Architecture}

Freeze-clamped samples were compared with samples frozen without compression. Two principles were followed in the histological evaluation of the integrity of tissue morphology.

(i) Ten to fifteen randomly selected sections from freeze-clamped samples were mixed with a similar number of sections from samples frozen without compression, and it was tested whether the two types of specimens could be distinguished upon histological examination. This was done for horizontal as well as vertical sections with both paraffin sections and frozen sections (see Methods). As demonstrated in Fig. 1 for CAB-stained paraffin sections, it was not possible to distinguish between compressed and noncompressed samples.

(ii) Horizontal as well as vertical sections from the two groups (compression and noncompression freezing) were examined systematically with respect to: (a) the distance from the surface of the liver (the capsule) at which positive identification of portal tracts could be carried out (i.e., identification of a bile duct and/or an artery in relation to a vein), and (b) the radial arrangement of the liver cell plates around a terminal hepatic vein as well as the pattern formed by terminal hepatic veins and portal tracts. No difference was noted between the two 
groups, neither in the series of horizontal sections, nor in the series of vertical sections. Furthermore, in both groups identification of portal tracts was possible at a distance of $100-200 \mu \mathrm{m}$ below the capsule of the liver. Since histological details (e.g., cell borders) are not as clearly seen in freeze sections as in paraffin sections, the latter part of the investigation was only carried out on the paraffin-mounted serial sections.

\section{The Efficiency of the Quenching of}

Metabolic Processes in Different Parts of the Sample

The geometry of the freeze-clamping tongs applied in the present study (1) will establish two essentially plane-freezing fronts in the sample, moving parallel to the surface toward the center (see Fig. 2). Thus, all parts of a section cut parallel to the surface can be expected to have experienced the same freezing time. Therefore, in order to identify the part of the freeze-clamped sample which has been frozen fast enough to preserve the in vivo metabolic state, the concentration of a number of labile metabolites were measured in a series of such parallel sections of a thickness of about $250 \mu \mathrm{m}$ (see Methods). Table 1 and Table 2 summarize the results of these experiments.

The data of Table 1 were obtained from livers cut out before freeze-clamping (see Methods) giving a delay of 5-7 s of circulatory interruption before the onset of freezing. Metabolite concentrations were measured in eight different samples of milling powder, representing concentration profiles perpendicular to the surface of the freezeclamped sample between 0.2 and $2.6 \mathrm{~mm}$. The control values, i.e., the " $0.2-\mathrm{mm}$ column," are in good agreement with what is usually found in freeze-clamped liver from pentobarbital-anaesthetized rats $(6-$ 8 ). In the second section (the " $0.5-\mathrm{mm}$ column"), ADP and AMP tend to rise, followed in the third section by a significant increase which is further augmented in deeper sections of the sample. A corresponding change is seen for ATP and for the calculated ATP/ADP ratio.

Since the data of Table 1 suggested that the metabolic state was altered in the major part of the sample, i.e., only the " $0.2-\mathrm{mm}$ sample" might be considered optimally frozen, another series of experiments were carried out with in situ freeze-clamping, i.e., without the delay caused by excision of the liver prior to freeze-clamping. The results are given in Table 2 . In these experiments, the linear resolution in the analysis of the metabolite concentration profiles are somewhat better since 10 sections are included over a distance of $2.3 \mathrm{~mm}$.

Compared with the data of Table 1, a number of differences may be noted: although not significantly different, the control values of Table 2 suggest a more optimal freezing with higher ATP/ADP ratio and a somewhat lower ADP and AMP. Furthermore the increase with depth of AMP and ADP is rather smaller, and a significant decrease in the calculated ATP/ADP ratio is seen only in section 9 and 10 while in Table 1 an increase was seen in section 3. Between 0.7 and $1.7 \mathrm{~mm}$ there is a transient significant increase in ATP with a corresponding increase in the adenylate sum. A similar transient increase in the adenylate sum is, however, also seen in Table 1 for the interval $0.9-1.6 \mathrm{~mm}$. The significance of this is not understood. The myokinase equilibrium expression does not change significantly with depth in any of the two series although there is a clear decreasing tendency, which is suggested also by the lower control value of Table 1, compared with Table 2 . In contrast, similar experiments on rat brain show a large increase of the adenylate kinase equilibrium expression $(9,10)$. The $P_{i}$ concentration (Table 2) remains constant for the first four sections after which there is a small rise amounting to $13 \%$ in the 10 th section. The calculated phosphorylation potential appears to be a 


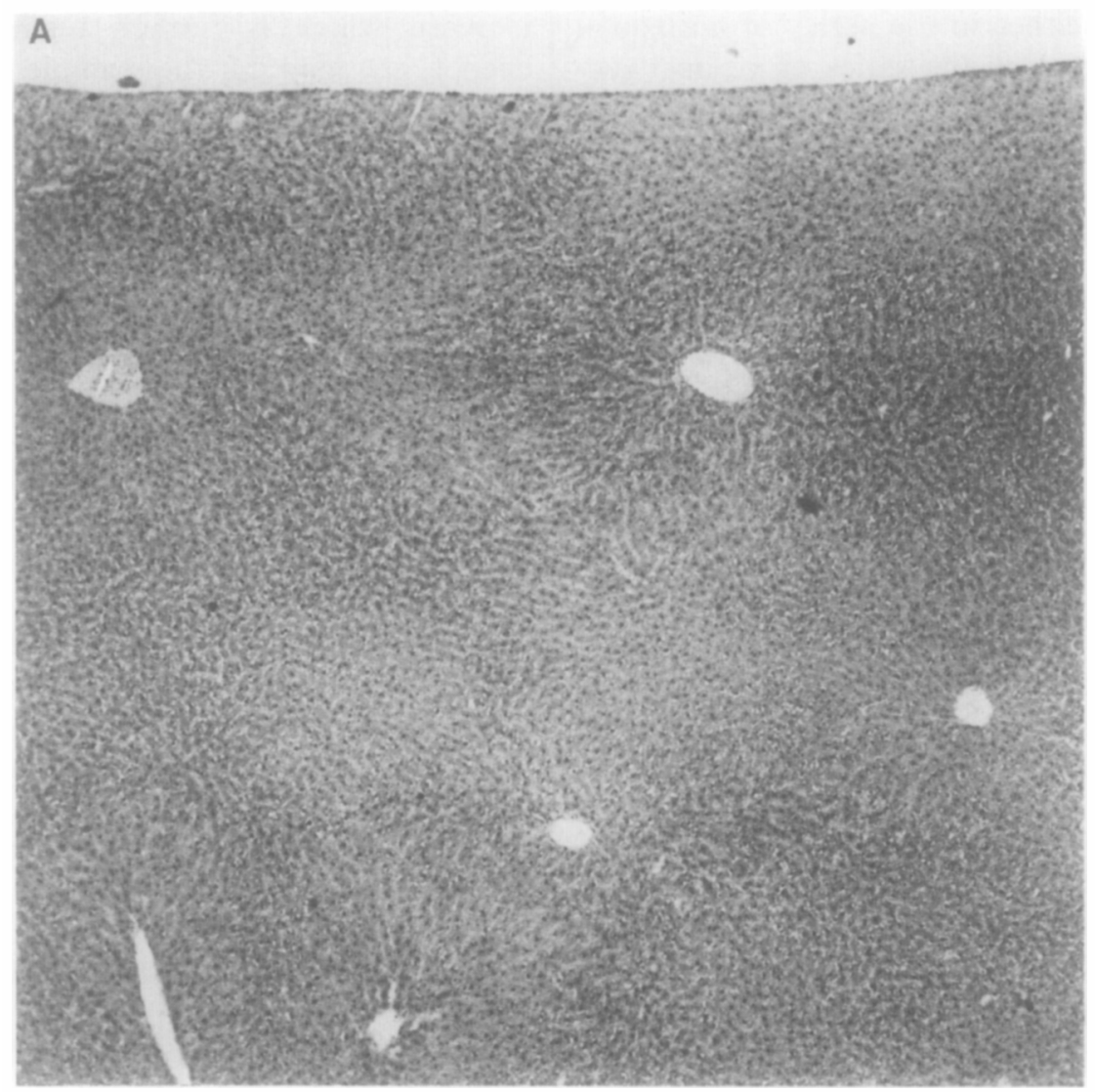

Fig. 1. Micrographs of rat liver. A liver lobe from a Pentothal-anaesthetized rat was excised and freeze clamped (compression freezing). Another lobe from the same animal was excised and frozen by being placed on an aluminum block precooled in liquid nitrogen, (noncompression freezing). Following fixation and paraffin mounting of the two samples, sections were cut perpendicular to the liver surface. The micrographs show parts of typical sections cut perpendicular to the liver capsule (at the top). Section (A) was taken from the lobe frozen without compression. Note the radial arrangement of the liver cell plates and the normal distribution of afferent and efferent veins. Section (B), taken from the freeze-clamped lobe, shows a similar picture with preserved radial arrangement of the liver cell plates and normal distribution of the veins. Sections were stained with CAB (3) $(\times 40)$.

most sensitive parameter of delayed freezing since a significant fall is seen already at a depth of $0.9 \mathrm{~mm}$, gradually increasing in the following sections.

In conclusion, the concentration profiles obtained for the in situ freeze-clamped samples (Table 2) all seem to display an initial plateau, suggesting this value reflects the actual in vivo metabolic state.

\section{DISCUSSION}

Two factors critically influence the reliability of the use of freeze-clamped samples for three-dimensional metabolic studies in vivo: first, the extent to which the tissue morphology in the freeze-clamped sample is preserved as compared with the in vivo state; second, the efficiency of the freezing, i.e., the size of the tissue volume of the frozen sample in which the metabolic state is fixed at the in vivo level.

\section{Morphological Aspects of Freeze-Clamping}

The liver acinus, or in some species, part of a few adjacent acini (forming a liver 


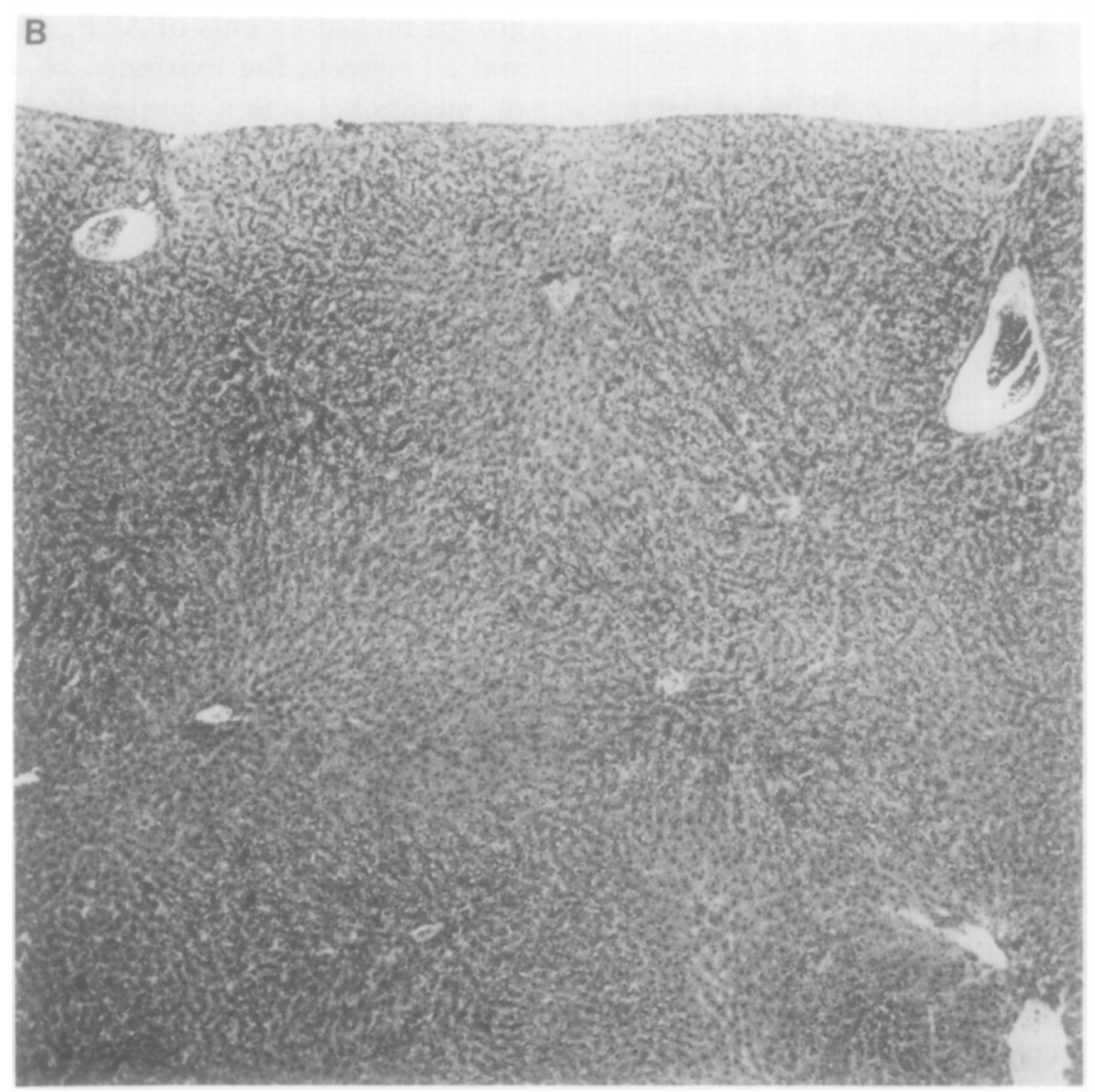

FIG. 1-Continued.

lobule), may be considered the functional unit of the organ (11). Consequently, a three-dimensional metabolic study of the liver would be concerned mainly with intralobular metabolic heterogeneity and with interlobular relations only for a small number of neighboring lobuli. Since the mechanical force applied during freezeclamping obviously changes the sample macroscopically when it is compressed to fill out the conical sample cup (see Fig. 2), the question asked in the present study was whether this macroscopic distortion of the edges was associated with a significant intra- and interlobular distortion in the central part of the frozen sample.

The histological study carried out showed that indeed a tissue block of approximately $6 \times 6 \times 2 \mathrm{~mm}$ was not significantly affected by the freeze-clamping (see Fig. 1).
The histological technique used in the evaluation of the morphological distortion involves a thawing either of the entire sample (paraffin sections) or of the individual sections (freeze sections) (2). Concerning the paraffin sections in particular, this procedure conceivably could allow a sample primarily distorted in the frozen state to resume near normal shape during thawing (sponge effect), and thereby obscure the histological diagnosis of distortion. However, the fact that distortion clearly can be detected in paraffin sections cut 4-5 $\mathrm{mm}$ closer to the edge of the sample, seems to indicate that such "sponge effect" does not occur.

\section{Efficiency of the Freeze-Stop}

The efficiency of freezing was evaluated by measuring the concentration of the 


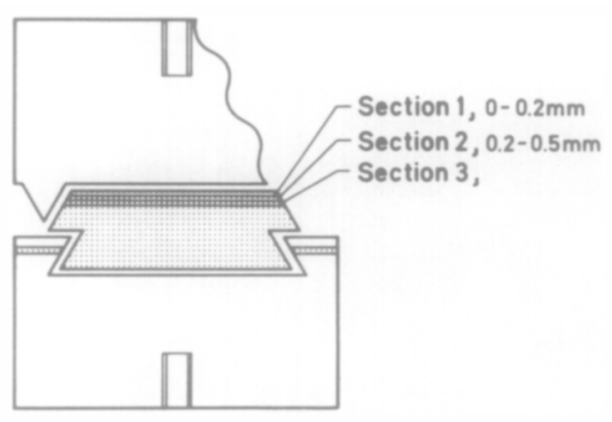

Fig. 2. Cross section of liver sample after freezeclamping. During freeze-clamping the liver lobe adapts the shape of the chamber formed between the two blocks of the freeze-clamping tongs, whereby the sample becomes rigidly fixed to the lower block. Parallel slabs of tissue were sampled for metabolite measurements by means of low-temperature milling in the frozen state (1).

adenine nucleotides as a function of distance from the surface of the frozen tissue block (cf. Fig. 2). Since the functional unit of the liver is much smaller than the volume of the single slab of tissue in which metabolite measurements were carried out, the changes observed with depth in the sample may be ascribed solely to the sampling procedure, i.e., provided the quenching of metabolic processes was fast enough, metabolite gradients would not be expected in the frozen sample.

Livers were freeze clamped either with or without a delay between circulatory arrest and onset of freezing, A and B samples, respectively (see Methods). In both groups measurements of ATP, ADP, AMP, and $P_{1}$ suggest the existence of a gradient of metabolic states perpendicular to the cooled surface, different, however, with respect to time course and point of departure. For the ATP/ADP ratio a steadily declining value starting at 4.4 may be observed in the A samples (Table 1), while the B samples clearly show an initial plateau at a value of 5.5, proceeding to a depth of about $1 \mathrm{~mm}$. ADP and AMP qualitatively follow this pattern. The existence of a zone of constant metabolic state near the surface in freeze-clamped samples has previously been demonstrated for perfused liver (16) and brain (17) by redox ratio measurements (18). The initial values of ATP, ADP, AMP, and ATP/ADP in the A samples (" $0.2-\mathrm{mm}$ column," Table 1) were 94, 118, 118, and $80 \%$, respectively, compared with the initial values of the B samples (Table 2). These figures compare well with those found in the B samples at a depth of $2.3 \mathrm{~mm}$, suggesting that the quenching time for that part of the sample should be similar to the 5- to 7-s delay of freezing for the A samples. In fact, the average freezing time ${ }^{2}$ for the 2.3-mm section may be calculated as ap-

${ }^{2}$ Freezing time $t$ defined as the time required for a temperature fall from $37-0^{\circ} \mathrm{C}$ at the particular distance $x$ from the cooled surface, calculated according to $t=k \cdot x^{2}(19)$. Experimentally $k$ has been determined to be $0.8-1.0$ with the applied freeze-clamping technique (1).

TABLE 1

Metabolite Concentrations Measured along the Freezing Gradient in Freeze-Clamped Rat Liver ${ }^{a}$

\begin{tabular}{|c|c|c|c|c|c|c|c|c|c|}
\hline \multirow[b]{3}{*}{ ATP } & \multicolumn{9}{|c|}{ Distance from surface $(\mathrm{mm})$} \\
\hline & \multicolumn{2}{|r|}{0.2} & 0.5 & \multirow{2}{*}{$\frac{0.9}{101=1}$} & \multirow{2}{*}{$\frac{1.2}{99 \pm 2}$} & \multirow{2}{*}{$\frac{1.6}{95 \pm 2}$} & \multirow{2}{*}{$\frac{1.9}{9 \pm 2 *}$} & 2.3 & 2.6 \\
\hline & 3.53 & \pm 0.11 & $101 \pm 1$ & & & & & $95 \pm 2 * *$ & $94 \pm 2 *$ \\
\hline ADP & 0.836 & \pm 0.060 & $109 \pm 6$ & $122 \pm 5^{* *}$ & $137 \pm 11^{* *}$ & $145 \pm 15^{* *}$ & $138 \pm 14^{*}$ & $133 \pm 8^{* *}$ & $139 \pm 9^{* *}$ \\
\hline AMP & 0.157 & \pm 0.015 & $103 \pm 8$ & $144 \pm 8^{*}$ & $146 \pm 14^{* *}$ & $162 \pm 18^{*}$ & $154 \pm 21^{*}$ & $145 \pm 14^{* *}$ & $152 \pm 13^{* *}$ \\
\hline$\sum \mathrm{AXP}$ & 4.51 & \pm 0.13 & $103 \pm 1$ & $105 \pm 1^{* *}$ & $108 \pm 1^{* *}$ & $106 \pm 2^{*}$ & $105 \pm 2$ & $104 \pm 2$ & $104 \pm 2$ \\
\hline ATP/ADP & 4.39 & 10.36 & $94 \pm 5$ & $84 \pm 3^{* *}$ & $75=5^{*}$ & $7 t \pm 6^{* *}$ & $73 \pm 6^{*}$ & $72 \pm 3^{* *}$ & $68 \pm 4^{* *}$ \\
\hline ATP $\cdot A M P / A D P^{2}$ & 0.86 & \pm 0.13 & $92 \pm 11$ & $87 \pm 5$ & $81 \pm 7$ & $78 \pm 10$ & $84 \pm 11$ & $84 \pm 11$ & $77 \pm 10$ \\
\hline
\end{tabular}

${ }^{a}$ A liver lobe from a Pentothal-anaesthetized rat was excised and freeze clamped within 5-7 s. Tissue powder from eight consecutive sections parallel with the surface of the frozen sample was obtained by low-temperature milling (1). The vertical distance from the surface of the frozen sample to the middle of each section is given in the top line of the table. The figures below represent metabolite concentrations in the different sections. The means of five experiments \pm SEM are given. The values measured in the first section, i.e., the "0.2-mm column," are expressed as $\mu \mathrm{mol} / \mathrm{g}$ wet $\mathrm{wt}$, while the following figures are percentages of these values.

* Significant difference with respect to " $0.2-\mathrm{mm}$ value" for $P<0.05(n=5)$.

** Significant difference with respect to " $0.2-\mathrm{mm}$ value" for $P<0.01(n=5)$. 
TABLE 2

Metabolite Concentrations Measured along the Freezing Gradient in Freeze-Clamped Rat Liver ${ }^{a}$

\begin{tabular}{|c|c|c|c|c|c|c|c|c|c|c|}
\hline & \multicolumn{10}{|c|}{ Distance from surface (mm) } \\
\hline & 0.2 & 0.5 & 0.7 & 0.9 & 1.3 & 1.5 & 1.7 & 1.9 & 2.1 & 2.3 \\
\hline ATP & $3.75 \pm 0.065$ & $102 \pm 1$ & $105 \pm 2^{* *}$ & $105 \pm 1^{* *}$ & $107 \pm 1^{* *}$ & $106 \pm 1^{* *}$ & $106 \pm 1^{* *}$ & $104 \pm 2$ & $100 \pm 1$ & $99 \pm 2$ \\
\hline ADP & $0.710 \pm 0.040$ & $102 \pm 4$ & $105 \pm 4^{\star}$ & $109 \pm 3^{* *}$ & $111 \pm 6$ & $107 \pm 8$ & $122 \pm 10$ & $116 \pm 6^{*}$ & $115 \pm 7^{*}$ & $118 \pm 5^{*}$ \\
\hline AMP & $0.133 \pm 0.042$ & $109 \pm 9$ & $106 \pm 4$ & $111 \pm 8$ & $115 \pm 7$ & $105 \pm 7$ & $117 \pm 5^{*}$ & $117 \pm 4^{*}$ & $106 \pm 7$ & $110 \pm 8$ \\
\hline$\sum \mathrm{AXP}$ & $4.59 \pm 0.070$ & $103 \pm 1$ & $105 \pm 2^{* *}$ & $106 \pm 2^{* *}$ & $108 \pm 2^{* *}$ & $106 \pm 1^{* *}$ & $109 \pm 2^{* *}$ & $105 \pm 2$ & $102 \pm 1$ & $102 \pm 2$ \\
\hline ATP/ADP & $5.5 \pm 0.59$ & $101 \pm 4$ & $100 \pm 3$ & $94 \pm 3$ & $98 \pm 6$ & $102 \pm 8$ & $89 \pm 7$ & $91 \pm 4$ & $87 \pm 5^{*}$ & $82 \pm 5$ \\
\hline \multicolumn{11}{|l|}{ ATP $\cdot$ AMP/ } \\
\hline $\mathrm{ADP}^{2}$ & $1.12 \pm 0.21$ & $111 \pm 10$ & $95 \pm 4$ & $94 \pm 3$ & $101 \pm 7$ & $88 \pm 12$ & $88 \pm 9$ & $89 \pm 7$ & $80 \pm 8$ & $83 \pm 11$ \\
\hline $\begin{array}{l}P_{\mathrm{i}} \\
\text { ATP/ }\end{array}$ & $2.97 \pm 0.12$ & $104 \pm 4$ & $95 \pm 4$ & $102 \pm 4$ & $109 \pm 5$ & $109 \pm 7$ & $114 \pm 11$ & $106 \pm 6$ & $108 \pm 5$ & $113 \pm 10^{*}$ \\
\hline$A D P \cdot P_{1}$ & $1860 \pm 195$ & $98 \pm 6$ & $106 \pm 4$ & $92 \pm 2^{*}$ & $90 \pm 5$ & $94 \pm 7^{*}$ & $79 \pm 7^{*}$ & $86 \pm 3^{* *}$ & $81 \pm 6^{*}$ & $74 \pm 7^{* *}$ \\
\hline
\end{tabular}

"A liver lobe from a Pentothal-anaesthetized rat was frecze clamped in situ. Tissue powder from 10 consecutive sections parallel with the surface of the frozen sample was obtained by low-temperature milling (1). The vertical distance from the surface of the frozen sample to the middle of each section is given in the top line of the table. The figures below represent metabolite concentrations in different sections. The means of five experiments \pm SEM are given. The values measured in the first section, i.e., the "0.2-mm column," are expressed as $\mu$ mol/g wet wt, while the following figures are percentages of these values.

* Significant difference with respect to the "0.2-mm value" for $P<0.05(n=5)$.

** Significant difference with respect to the "0.2-mm value" for $P<0.01(n=5)$.

proximately $5 \mathrm{~s}$. Similarly for the surface section, the average freezing time is about $0.1 \mathrm{~s}$. Thus, the difference of about $5 \mathrm{~s}$ between these two figures compares well with the recorded delay of freezing of the excised samples and could explain the differences of metabolite concentrations observed with the two methods of sampling.

In conclusion, the data of Tables 1 and 2 suggest that in situ freeze-clamping of rat liver with the technique applied in the present study preserve the in vivo metabolic state in the part of the sample within a distance of about $1 \mathrm{~mm}$ from the tissuemetal interface. At a distance of $2 \mathrm{~mm}$, changes of 10-20\% are observed for ADP, AMP, and $\boldsymbol{P}_{\mathrm{i}}$. For samples freeze clamped with a delay of 5-7 s, the changes induced by the sampling procedure are considerably larger, e.g. , at a depth of $2 \mathrm{~mm}$, on the order of 60-80\% for ADP, AMP, and ATP/ADP compared with the initial values of the in situ frozen samples.

\section{ACKNOWLEDGMENTS}

The authors wish to thank Mrs. Lissi Immerdal, Department of Biochemistry, and Anne-Marie Mikkelsen and Hans Stilling Petersen, Department of Pathology, for valuable technical assistance.

\section{REFERENCES}

1. Quistorff, B., and Chance, B. (1980) Anal. Biochem. 108, 237-248.

2. Poulsen, H., and Christoffersen, P. (1979) Atlas of Liver Biopsies, pp. 14, 219-222, Munksgaard and Lippencott, Copenhagen/Philadelphia.

3. Lillie, R. D. (1940) Stain Technol. 15, 17-22.

4. Lowry, O. H., and Passonneau, J. V. (1972) A Flexible System of Enzymatic Analysis, pp. 124, 148, 152, Academic Press, New York.

5. Schultz, D. W., Passonneau, J. V., and Lowry, O. H. (1967) Anal. Biochem. 19, 300-314.

6. Dale, R. A. (1965) J. Physiol. 181, 701-711.

7. Reinauer, H., and Hollmann, S. (1966) Anaesthetist 15, 327-332.

8. Seitz, H. J., Faupel, R. P., Kampf, S. C., and Tarnowski, W. (1973) Arch. Biochem. Biophys. 158, $12-18$.

9. Quistorff, B. (1980) in Cerebral Metabolism and Neuronal Function (Passonneau, J. V., Hawkins, R. A., Welsh, F., and Lust, W. D., eds.), Williams \& Wilkins, Baltimore, in press.

10. Kobayashi, M., Lust, W. D., and Passonneau, J. V. (1977) J. Neurochem. 29, 53-59.

11. Rappaport, A. M. (1976) Beitr. Pathol. Bd. 157, 215-243.

12. Brosnan, J. T., Krebs, H. A., and Williamson, D. H. (1970) Biochem. J. 117, 91-96.

13. Hems, D. A., and Brosnan, J. T. (1970) Biochem. J. 120, 105-111.

14. Bücher, Th., Krejci, K., Rüssmann, W.. Schnitger, H., and Wesemann, W. (1964) in Rapid Mixing and Sampling Techniques in Biochemistry (Chance, B., Eisenhardt, R. H., Gibson, 
Q. H., and Lonberg-Holm, K. K., eds.), pp. 255-264. Academic Press, New York.

15. Faupel, R. P., Seitz, H. J., Tarnowski, W., Thiemann, V., and Weiss, CH. (1972) Arch. Biochem. Biophys. 148, 509-522.

16. Quistorff, B., Chance, B., and Takeda, H. (1978) in Frontiers of Biological Energetics (Dutton, P. L., Leigh, J. S., and Scarpa, A., eds.), Vol. 2, pp. 1487-1497, Academic Press, New York.
17. Quistorff, B., and Chance, B. (1976) in Oxygen and Physiological Function (Jöbsis, F. F., ed.), pp. 100-110, Professional Information Library, Dallas, Tex.

18. Chance, B., Schoener, B., Oshino, R., Itshak, F., and Nakase, Y. (1979) J. Biol. Chem. 254(11), 4764-4771.

19. Wollenherger, A., Ristau, O., and Schoffa, G. (1960) Pflügers Árchiv. 270, 399-412. 